\title{
La comunidad escolar como medio para el desarrollo socio emocional de los alumnos. Un estudio de caso en la República Argentina
}

\author{
The school community as a means for the social emotional development \\ of the students. A case study in the Argentine Republic
}

\author{
Carolina Sánchez Agostini ${ }^{a}$, Florencia Teresita Daura, \\ Julieta Laudadio ${ }^{c}$ \\ ${ }^{a}$ Facultad de Ciencias Biomédicas. Escuela de Educación. Universidad Austral. \\ csanchez@austral.edu.ar \\ ${ }^{b}$ CONICET, Universidad Austral. \\ fdaura@austral.edu.ar \\ ${ }^{c}$ Facultad de Filosofía, Humanidades y Artes. \\ Instituto de Investigaciones en Educación en las Ciencias Experimentales. \\ Universidad Nacional de San Juan. \\ jlaudadio@ffha.unsj.edu.ar
}

\section{RESUMEN}

La educación socio emocional ha cobrado una relevancia social y científica notable en los últimos años. El objetivo de este artículo es describir la percepción de estudiantes, personal docente y directivo, a fin de analizar la función de la escuela como ámbito facilitador de conductas promotoras de la educación socioemocional. La muestra utilizada fue de 134 estudiantes, 23 docentes y directivos de un colegio secundario de gestión estatal de la provincia de San Juan, Argentina. Se utilizó el cuestionario de Lickona y Davidson (2003) School as Caaring Community Profile-II para evaluar percepciones de respeto, amistad y pertenencia, configuración de su medioambiente, soporte y atención por parte del personal docente y de los alumnos, también en relación a las familias. Los resultados arrojaron que predomina una percepción positiva, en la Institución, sobre el soporte brindado tanto por los padres de familia como por la misma Institución. Se brindan recomendaciones para consolidar el papel de la escuela.

Palabras claves: educación socio emocional, adolescencia, escuela, familia.

\footnotetext{
ABSTRACT

Socio-emotional education has gained significant social and scientific relevance in recent years. The aim of this article is to describe the perception of students, teachers and principals, in order to analyze the role of the school as a facilitator of behaviors that promote socio-emotional education. The sample was 134 students, 23 teachers and directors of a state-run secondary school in the province of San Juan, Argentina. We used the questionnaire of Lickona and Davidson (2003) School as Caaring Community Profile-II to evaluate perceptions of respect, friendship and belonging, configuration of their environment, support and attention by teachers and students, also in relation to the families. The results showed that a positive perception predominates, in the Institution, on the support provided by both the parents and the Institution itself. Finally, there are recommendations to consolidate the role of the school.

Key words: socio emotional education, adolescence, school, family.
} 


\section{INTRODUCCIÓN}

El análisis de la comunidad escolar como medio para el desarrollo socioemocional de los alumnos se considera de gran relevancia para la prevención de diversos conflictos tanto personales como sociales (Berger et al., 2009; Clouder et al., 2013; entre otros).

Las sociedades están compuestas por los aportes que cada uno de los ciudadanos realiza. Teniendo en cuenta que la función social de las escuelas es la de contribuir a la cultura, a enseñar a vivir juntos, a compartir con los demás y, por tanto, a desarrollar la socialización (Çınkır, Nayir \& Çetin, 2016), puede verse de qué manera el estudio de esta temática en los ámbitos educativos adquiere una gran importancia.

La carencia de una educación integral trae consecuencias negativas para los jóvenes tales como adicciones, bullying, estrés, baja autoestima, ansiedad, depresión, y conflictos en la convivencia que repercuten también en su rendimiento escolar o académico y que podrían prevenirse con una educación más comprensiva de todas las dimensiones de la persona (Bernal, González-Torres \& Naval, 2015).

La investigación psicológica y educativa, cuenta con importantes avances en los últimos tiempos, sin embargo, la comprensión científica acerca del funcionamiento emocional y de los factores promotores o de riesgo, están aún lejos de ser comprendidos en profundidad (Bauman, 2003 en Craig, 2009). Puede verse así la relevancia del estudio y la investigación en esta materia.

La escuela puede convertirse en una promotora de interacciones saludables o bien en un marco en el que las conductas de violencia son, al menos, aceptadas. Esto impacta de una manera muy directa en el desarrollo socioemocional de niños y adolescentes y de la sociedad en general ya que, como afirman Bernal, González-Torres y Naval (2015), las metas vitales de cualquier niño (bienestar y felicidad, ajuste social, amigos, ocio saludable, rendimiento escolar, etc.) son más asequibles si tiene oportunidad de acceder a una educación que abarque a todas sus capacidades.

Frente a este planteo, surgen algunos interrogantes que en el ámbito de la presente investigación intentamos responder ¿Cómo perciben el contexto educativo los actores de una institución educativa? ¿Existen diferencias al respecto en función de la edad de los actores institucionales? ¿Cómo interactúa en esta percepción el rol que cada actor desempeña dentro de la institución?

A partir de estas preguntas se plantearon los siguientes objetivos. Por un lado, describir la percepción que los alumnos y el personal docente y directivo de un colegio secundario de gestión estatal de la provincia de San Juan (Argentina) tienen sobre la cultura solidaria que en esta predomina; por el otro, de acuerdo con los resultados obtenidos, brindar orientaciones dirigidas a promover este aspecto de la educación integral.

De acuerdo con esto, en primer lugar, se detalla un breve marco teórico sobre la educación socioemocional, sobre el papel que los padres tienen en el desarrollo socioemocional de los alumnos y sobre el rol que al respecto le compete a la escuela. En segundo lugar, se describe el diseño metodológico adoptado, de carácter cuantitativo, que se desarrolló con una muestra no probabilística, seleccionada por conveniencia, perteneciente a una institución estatal e integrada por 157 sujetos, que completaron el Cuestionario School as Caaring Community Profile-II.

Por sus características, se trata de un estudio que se destaca por su originalidad en tanto que, si bien son numerosas las investigaciones y experiencias centradas en la promoción de 
la educación socioemocional ${ }^{1}$ en población argentina (De Fabritis, julio de 2018; Paneiva Pompa, Bakker y Rubiales, 2016; Mikulic, 2013), esta se trata de la primera investigación formal realizada en la ciudad mencionada y que presenta datos enriquecedores a partir de la utilización del cuestionario de Lickona y Davidson (2003).

\section{LA EDUCACIÓN SOCIOEMOCIONAL}

El abordaje de esta temática conlleva profundizar algunos conceptos o constructos que lo entrecruzan; particularmente, la regulación emocional, la educación emocional, la inteligencia emocional y la educación social.

El primero de estos, la regulación emocional, que permite controlar los impulsos y las emociones, es el logro psicosocial más relevante entre los 2 y los 6 años (Eisenberg et al., 2004, en Berger, 2016). Su florecimiento se complementa con otra adquisición importante que, según Berger, ocurre durante la segunda infancia: el desarrollo de la moralidad y sus respectivos valores. Esta etapa también es crucial para el desarrollo de la autoestima y el autoconcepto, que sentará las bases para transitar la adolescencia, etapa en la que la identidad, las relaciones y las emociones pasan por la crisis propia del desarrollo que pueden llevar a futuras complicaciones si no se logran atravesar adecuadamente.

Precisamente, el dominio de las emociones es parte normal del desarrollo psicosocial del niño, sobre todo en los siete primeros años de vida. En efecto, los niños que no aprenden los límites de una conducta aceptable, pueden desarrollar problemas emocionales moderados o graves, entre ellos comportamientos anormales y trastornos de personalidad (Craig, 2009).

Estas ideas permiten considerar la importancia de educar la emocionalidad de cada niño, siendo necesario delimitar qué se comprende por educación emocional. Sobre esta, a grandes rasgos podemos decir que es la que permite orientar el desarrollo evolutivo del niño y del adolescente según cada etapa. No obstante, ahondando en la literatura, encontramos que Trianes Torres y García Correa (2002, p. 6), sostienen que se trata de un "proceso educativo, continuo y permanente que pretende potenciar el desarrollo emocional como complemento indispensable del desarrollo cognitivo, constituyendo ambos los elementos esenciales del desarrollo de la personalidad integral". A estas dos dimensiones se agregan la social y espiritual, que permiten que el educando se trascienda a sí mismo, realice aportes creativos y/o enriquecedores en el contexto que lo rodea y conviva con otros. Por lo que, el fin de la educación emocional, en conjunto con las otras dimensiones educables de la persona, es capacitar a los estudiantes para los desafíos que plantea la vida cotidiana con el objeto de aumentar el bienestar personal y social, ayudándolos a autoconocerse y consolidar su proyecto de vida.

El tercer constructo que se vincula con los anteriores conceptos es el de inteligencia emocional, estudiado por numerosos autores (Bar-On, 1997; Salovey y Mayer, 1990; Shapiro, 1997) y popularizado por Goleman a través de su libro homónimo (Goleman, 2012), que consiste en el uso inteligente de las emociones (Alterio Arriola y Pérez Loyo,

Entre los programas desarrollados para promover la educación socioemocional podemos mencionar: Programa de Educación Emocional en el Colegio Washington School; "Sin Afecto no se Aprende ni se Crece. Un programa para fortalecer los recursos afectivos, cognitivos y lingüísticos"; Programa Clima Emocional Positivo en el Aula (CEPA) (en Mikulic, 2013); el Hero Program, desarrollado por Mesurado, Di Stéfano y Robiolo (Mesurado, Distefano, Robiolo y Richaud, 2018; Hero, 2016). 
2005). Efectivamente, es aquella que permite guiar el comportamiento y la expresión de la emoción.

En su abordaje se comprende que la inteligencia está ligada tanto a lo cognitivo como lo emocional y, en consecuencia, que el proceso de aprendizaje no sólo depende del conocimiento, sino también de cómo el alumno controla sus emociones para su propio beneficio y el de las personas que lo rodean. Los autores proponen cinco áreas fundamentales de habilidades en las que se comprende y desarrolla la inteligencia emocional: autoconocimiento emocional, control emocional, automotivación, reconocimiento de las emociones ajenas y habilidad para entablar relaciones interpersonales.

Por último, la educación de la dimensión social se desprende del ser relacional propio del hombre, de su tendencia natural a vincularse con otras personas para desarrollarse, satisfacer necesidades, enriquecerse y enriquecer a los demás; por ser un ciudadano que necesita vivir con otros en tres ámbitos (Barrio Maestre, 2016): consigo mismo, con sus familiares, con los demás ciudadanos. La educación de esta dimensión, por tanto, se dirige a desarrollar en el hombre esta tendencia natural a vivir con otros, a ser un ciudadano reflexivo y dialógico (Barrio Maestre, 2016) capaz de modificar en forma constructiva, creativa y cierta la sociedad en la que reside, buscando el beneficio propio y el de las personas con las que convive.

Llegamos así al momento de hacer referencia a la educación socioemocional, que en síntesis conlleva promover el desarrollo de la dimensión emocional o volitivo-afectiva y social del alumno. De allí es que, al momento de plantearla, resulta importante no sólo establecer cuáles serán los objetivos, las competencias o capacidades, los contenidos, y los recursos a desarrollar sino, principalmente, cuál es el contexto desde el que se la abordará y cuáles son los factores que pueden favorecerla o no.

Entre los elementos que se mencionan, los objetivos delimitan el fin hacia el cual tiene que dirigirse la educación socioemocional. Montoya et al. (2016) plantean algunos de carácter general que pueden ser considerados en las propuestas que se diseñen: ayudar a conocer las emociones y las reacciones emocionales como forma de potenciar las positivas y frenar las negativas; fomentar el pensamiento reflexivo y divergente en el proceso de afrontamiento y resolución de situaciones problemáticas; educar para la participación social; educar en los principios de cooperación y de trabajo en equipo; lograr la capacidad de ponerse en el lugar de los otros, respetando los distintos puntos de vista; conocer y poner en práctica los principios argumentales para la defensa de las ideas propias, sin menospreciar las ideas contrarias; fomentar la implicación del alumnado en el proceso de enseñanza-aprendizaje, a través de la motivación y la reflexión; y fomentar la resolución de problemas de forma creativa y dotar estrategias que faciliten la solución eficaz de los mismos.

En relación con las competencias ${ }^{2}$ que pueden desarrollarse a través de la educación socioemocional y que suponen objetivos y contenidos; Bernal, González-Torres y Naval (2015) hacen referencia a las competencias de la inteligencia emocional y afirman que guardan vinculación con algunas descripciones del carácter: la autoconsciencia, la consciencia social, el autogobierno y la organización; y la gestión.

En el contexto de este trabajo utilizamos como sinónimos los conceptos de competencia, capacidad y virtud o fortaleza de carácter, eludiendo el tratamiento de las diferencias epistemológicas que existen entre estos conceptos. $\mathrm{Al}$ respecto, invitamos a profundizar la obra de Amilbiru (2015). 
Del mismo modo, Peterson y Seligman (2004) explican que las fortalezas de carácter son características o disposiciones positivas que tiene o llega a desenvolver una persona y que se reflejan a través de sus pensamientos, sentimientos y comportamientos, contribuyendo en forma positiva en su desarrollo (Park, Peterson \& Seligman, 2004). Profundizando su estudio, en un libro que ya es clásico, Peterson y Seligman (2004) presentan una clasificación de 24 fortalezas de carácter: amor, amor por el aprendizaje, apreciación de lo bello, autenticidad, autorregulación, bondad, civismo, creatividad, curiosidad, esperanza, espiritualidad, imparcialidad, inteligencia social, gratitud, humor, liderazgo, modestia, pensamiento crítico, perdón, perseverancia, prudencia, sensatez, valentía y vitalidad.

En relación con los contenidos Rodríguez Fernández y Linares Von Schmiterlow (2002) afirman que enseñarlos implica pensar, sentir y actuar sobre la cultura. De esta manera, introducen el término de "cultura pensada" y ponen de relevancia la necesidad de que el currículum haga referencia directa al carácter social y ético que tiene la formación de los estudiantes.

Precisamente, en relación con "pensar" los contenidos que se pueden abordar en el proceso de educación socioemocional, son varios los autores que en los últimos quince años describieron diversas propuestas. Entre ellos, Rodríguez Fernández y Linares (2002) proponen los contenidos que debe incluir una propuesta educativa en materia de enseñar a convivir, entre los que se cuentan: adecuación a los cambios sociales, a la sociedad multicultural y al contexto familiar; desarrollo de habilidades sociales y distintas competencias como el diálogo, el trabajo en equipo, la flexibilidad y la participación; la coordinación entre el profesorado, los directivos; promover valores como la tolerancia, la colaboración y la responsabilidad, entre otras.

Lickona y Davidson (2003), por su parte, sostienen que los contenidos tienen que estar dirigidos a desarrollar virtudes, partiendo de la base de que "el contenido del buen carácter" es la virtud. Vemos ya en su postura la introducción de fortalezas de carácter o de virtudes (Peterson y Seligman, 2004) que son necesarias en el desarrollo socioemocional del educando. Es así como propone diez virtudes esenciales para promover un "buen carácter", al que podríamos llamar socioemocional o equilibrado: sabiduría, justicia, fortaleza, autocontrol, amor, actitud positiva, trabajo duro, integridad, gratitud y humildad.

Montoya et al. (2016) formulan cuatro bloques de contenidos que pueden abordarse a través de la educación emocional: 1. Conocimiento y gestión de las emociones. Educación de las emociones; 2. La autorregulación de las emociones y autonomía personal; 3. Habilidades de comunicación: inteligencia emocional y contextual; 4. Habilidades de afrontamiento eficaz y asentamiento social.

Echeita (2016) plantea un marco de valores incluyentes que deberían organizar la convivencia y la forma de vivir juntos: equidad, comunidad, derechos, participación, coraje, sostenibilidad, esperanza, compasión, alegría, no violencia, respeto por la diversidad, amor, honestidad, sabiduría, confianza y belleza.

En relación con los factores que pueden favorecer o no la educación socioemocional, Bernard (2004) propone algunos que han tenido gran influencia en la orientación práctica del desarrollo juvenil, distinguiendo entre factores internos y externos. Los primeros hacen referencia a las características personales del individuo que hablan de su capacidad para responder positivamente a las exigencias del ambiente y, los segundos, ponen su foco en el ambiente como favorecedor de estos factores internos, como el soporte afectivo, la comunidad, las exigencias del ambiente. 
Entre los factores externos podemos considerar lo señalado por Berkowitz (2018) con respecto a la importancia de contar con referentes a la hora de educar el carácter en sus dimensiones cognitivas, afectivo-motivacionales y comportamentales. En este sentido, afirma que detrás de cada fortaleza de nuestro modo de ser hay una persona que confió y esperó más de nosotros, sembrando una motivación para superarnos. Asimismo, asevera que la educación del carácter implica desarrollar un modo de ser, y empezamos a ser junto a otros; padres y docentes son los referentes cercanos, el espejo en el que se miran los niños y jóvenes esperando encontrar en ellos las actitudes que se les exigen. De allí la importancia de construir vínculos saludables tanto en el ámbito familiar como en el ámbito escolar.

Los factores externos, sin duda, integran un aspecto que posee gran importancia en la educación socioemocional y al que hicimos referencia: el contexto en el que se desarrolla el proceso educativo. Efectivamente, para un aprendizaje positivo, es relevante el clima que se genera a partir de las relaciones interpersonales y de los valores que se experimentan mediante las relaciones. De esta manera, la escuela y la familia se presentan como actores fundamentales en dicha educación; su accionar se fortalece y acrecienta si ambos buscan los mismos objetivos respecto al desarrollo socioemocional de los alumnos (Naval, Bernal y Fuentes, 2018).

Este planteamiento nos lleva a considerar cuál es el rol que le compete a los padres y la institución educativa en el desarrollo socioemocional, cuestiones que abordamos en los siguientes apartados.

\subsection{EL PAPEL DE LOS PADRES EN EL DESARROLLO SOCIOEMOCIONAL}

Encontramos investigaciones (Cuervo Martínez, 2010; Cáceres Muñoz, 2015; Henao López \& García Vesga, 2009; Márquez-Cervantes \& Gaeta-González, 2018) que analizan la relación entre las pautas y los estilos de crianza y el desarrollo socioafectivo durante la infancia, las mismas asumen que la salud mental de los padres, las pautas de crianza y el desarrollo socioafectivo de los niños están muy relacionados. Entre sus conclusiones destaca la importancia de la familia para facilitar el desarrollo de conductas prosociales y la autorregulación emocional y para la prevención de problemas de salud mental como depresión, agresividad, baja autoestima y ansiedad, entre otras (Cuervo Martínez, 2010).

Antes del nacimiento, el niño participa del estado emocional de la madre y del ambiente en el que se encuentra. Su manera de comunicarse es básicamente a nivel sensible, su primer contacto con la realidad es afectivo. Y a medida que va creciendo necesita tanto el consuelo y la ternura de la madre, como la exigencia y fortaleza del padre. Se trata de la complementariedad de los padres en la educación socioafectiva de los hijos, ya que es más propio del padre ayudar al hijo a enfrentarse con los retos, con los problemas, y de la madre ser acogedora y dar consuelo ante el dolor o las dificultades que se presentan en la vida (Castillo Córdova, 2013).

Un estudio más reciente (Hernández-Veloz, Gaeta-González y García-Gordillo, 2016) analiza la relación entre variables socio-familiares como el tipo de familia y el nivel académico de los padres; los aspectos afectivo-motivacionales que inciden en el desarrollo académico de los adolescentes, particularmente el planteo de metas académicas y el tipo de motivación que en ellos predomine (de aprendizaje o de rendimiento) (Dweck y Leggett, 1988; Nicholls, 1978, 1984, 1989). Las investigaciones también señalan que alumnos de 
familias nucleares tienen mejor manejo emocional y metas de aprendizaje superadoras, en comparación con los alumnos de familias monoparentales. Del mismo modo, el nivel académico de la madre se relaciona significativamente con las emociones positivas y las metas de aprendizaje de los hijos.

Márquez Cervantes y Gaeta González (2016) reflexionan acerca del trabajo de los padres en la educación afectiva y sostienen que estos brindan dos tipos de escudos en la vida de los hijos. Uno para protegerlos físicamente, cubriendo necesidades básicas tales como alimento, vestido, salud, etc.; y otro para protegerlos emocionalmente, donde se deben cubrir necesidades emocionales como sentirse querido, admirado, respetado, reconocido, aceptado, etc. Así afirman que la educación afectiva está encaminada a satisfacer estas necesidades y a enseñarles cómo desarrollar estos aspectos en su propia vida. De allí la importancia de que los padres reflexionen sobre cómo interactúan personalmente con sus hijos, su estilo educativo, cómo manejan sus propias emociones y cómo suelen responder a las diversas reacciones emocionales de otras personas. Conocer y gestionar adecuadamente las propias emociones, es vital para promover el bienestar físico y emocional personal y de los demás.

Los antecedentes teóricos y empíricos evidencian la importancia que tiene el papel de los padres y madres de familia como referentes en el desarrollo emocional de sus hijos, principalmente para ayudarlos a tener más seguridad de sí (Cuervo Martínez, 2010; Cáceres Muñoz, 2015; Henao López \& García Vesga, 2009; Márquez Cervantes y Gaeta González, 2018). Al mismo tiempo, señalan que la comunicación entre padres e hijos, así como la disciplina y la toma responsable de decisiones son aspectos que requieren de un mayor trabajo. Entre otros aportes que estas investigaciones brindan, se encuentra el énfasis en la necesidad de que los padres, como responsables primarios de la educación de la familia, tomen consciencia de las propias emociones y trabajen sobre ellas a fin de lograr una estabilidad emocional y una vida plena en todos sus integrantes. Y se advierte como contradictorio que los padres deseen que sus hijos tengan una vida exitosa, sana y feliz; cuando ellos mismos no tienen capacidad para generar un clima afectivo, motivador y emocionalmente equilibrado.

\subsection{EL PAPEL DE LA ESCUELA EN EL DESARROLLO SOCIOEMOCIONAL}

El tema de la convivencia escolar ha cobrado una visibilidad muy significativa y se ha constituido en un tema prioritario en la agenda de las políticas educativas. Del mismo modo, se constata que existe un grupo importante de alumnos que no están satisfechos con el clima escolar y con las maneras de actuar de los docentes y que existe un bajo nivel de diálogo entre las instituciones educativas y las familias (Beech y Marchesi, 2008).

Parece fundamental colocar estas cuestiones en el marco de una estrategia donde aprender a vivir juntos constituya un eje central de las experiencias de aprendizaje que los alumnos desarrollan en las escuelas. El clima que a partir de esta estrategia se genere en la institución es por ello muy importante.

Al respecto, Beech y Marchesi (2008) afirman que la convivencia consiste, en gran medida, en compartir; y a compartir se aprende: a compartir tiempos y espacios, logros y dificultades, proyectos y sueños. Por ello, estos mismos autores (Beech y Marchesi, 2008) sostienen que el aprendizaje de valores y de habilidades sociales, de los que se desprenden las buenas prácticas de convivencia, son la base del futuro ciudadano. 
Ahondando en los aspectos que actualmente regulan las escuelas Litichever (2012) explora aquellos que están incluidos en las normativas de convivencia a fin de identificar la existencia de un marco regulatorio compartido. Entre los resultados señala que las normas se vinculan con la apariencia, las relacionadas con la puntualidad; las que hacen referencia al cuidado de la institución; las que se refieren al respeto de los símbolos patrios o rituales escolares; las que se concentran sobre asuntos más puntuales del proceso de enseñanzaaprendizaje y, en ocasiones, también se explicitan normas que buscan regular la relación con los otros. Al respecto concluye que sería deseable que dichas pautas reflejaran la necesidad de enseñar a convivir en el ámbito escolar y social.

Las ideas expresadas por Litichever (2012) afianzan el interés vigente en el ámbito de la enseñanza en general de lograr objetivos educativos de alto nivel, encaminados a formar integralmente a los estudiantes; esto es, tanto en su dimensión intelectual como en la afectiva. Por lo que se enfatiza en la relevancia del desarrollo emocional en vistas a contribuir a su bienestar y equilibrio afectivo (Gaeta y López, 2013). De acuerdo con esto, es necesario seguir dando pasos para contar con investigaciones que aborden el desarrollo socioafectivo de los estudiantes y los factores que lo favorecen.

En esta línea, Martínez-Garrido y Murillo (2015) exploran algunos factores educativos que impactan sobre el autoconcepto de los estudiantes, identificando cinco aspectos: la experiencia docente, la evaluación de los aprendizajes, la metodología de enseñanza, la atención a la diversidad y maximizar el uso del tiempo en el aula.

Asimismo, Garrido Natarén y Gaeta González (2016) señalan la importancia que tiene el rol del docente en la promoción del aprendizaje de los alumnos y del desarrollo socioemocional que este proceso conlleva; insistiendo que para esto es necesario profundizar el estudio sobre los estados socioemocionales del docente para que los profesionales de la enseñanza puedan desenvolverse adecuadamente en el aula. De acuerdo con esto, apuntan a la trascendencia de desarrollar la capacidad para percibir emociones; de acceder, generar y utilizar las emociones para ayudar al pensamiento, y regular las emociones a fin de promover el crecimiento emocional e intelectual.

Gaeta González y Martínez Otero-Pérez (2017) nos presentan algunas reflexiones y experiencias empíricas en relación con el desarrollo emocional en diferentes contextos educativos. Entre sus aportes señalan a la adolescencia como una etapa de fragilidad emocional en la que es fundamental la educación de la afectividad, ya que los cambios biológicos, cognoscitivos, psicosociales y emocionales, conforman una compleja trama que nos permite entender mejor por qué en la búsqueda de una nueva identidad, es habitual que el adolescente cambie de ánimo, experimente una intensa emotividad o ponga constantemente a prueba los límites como modo de reafirmar su independencia y autonomía. De esta manera se presenta esta etapa como una oportunidad para educar emocionalmente a los adolescentes, previniendo comportamientos disruptivos y sus consecuencias perjudiciales. Entre las pautas que orientan el desarrollo emocional en los jóvenes, se enfatiza en primer lugar la necesidad de educar emocionalmente a los adultos significativos -como padres o profesores-, para ampliar las posibilidades de realizar con mayor éxito su tarea educativa (Márquez Cervantes, 2017).

Márquez Cervantes y Gaeta González (2018) sostienen que para que el alumno logre desarrollar planamente su dimensión afectiva es clave contar con un "educador emocional". El docente, además de poseer los conocimientos de la materia a impartir, debe ser capaz de transmitir una serie de valores a sus alumnos, trabajar sobre los procesos que faciliten 
la toma de decisiones con responsabilidad y ha de ser una figura mediadora. Es decir, que debe ser un ejemplo a seguir, ya que los alumnos pasan en las aulas gran parte de su infancia y adolescencia, periodos en los que se produce, principalmente, su desarrollo emocional.

La práctica de la educación afectiva implica, por tanto, diseñar programas fundamentados en un marco teórico; y para llevarlos a la práctica, contar con un profesorado preparado y bien dispuesto. Para esto, es importante que el docente se convierta en modelo de afrontamiento emocional, de habilidades empáticas y de resolución reflexiva de conflictos interpersonales, como fuente de aprendizaje para sus estudiantes. En virtud de lo anterior, el desarrollo emocional requiere de un trabajo continuo que inicia en la infancia, se desarrolla en la adolescencia y se define en la edad adulta, por lo que requiere de personal educativo emocional que, a su vez, sea un modelo de afrontamiento emocional y de habilidades interpersonales (Reina Flores y Oliva, 2015).

Al respecto Márquez-Cervantes y Gaeta-González (2018) señalan la importancia de que los docentes favorezcan un trabajo en el aula para el reconocimiento de las emociones y el fortalecimiento de las relaciones interpersonales positivas entre los alumnos; como así también la toma responsable de decisiones. De esta forma, el ambiente del aula se configura como un espacio idóneo de socialización emocional, donde los docentes con sus actitudes y comportamientos constituye el principal referente.

El clima en el aula influye notablemente en la actitud del profesor y de los alumnos hacia la enseñanza y el aprendizaje. Un clima de confianza y respeto mutuo favorece tanto el desarrollo de los alumnos como la actividad docente. Algunas de las conductas específicas de los profesores para crear un ambiente favorable de aprendizaje son: potenciar actitudes positivas de los alumnos hacia la temática a abordar, mantener a pesar de posibles dificultades expectativas positivas hacia los alumnos, ofrecer apoyo y ánimo a quienes tienen dificultades, enfrentar con calma los posibles inconvenientes que surjan en el aula, trasmitir una preocupación personal por cada alumno y su aprendizaje y favorecer la confianza de los alumnos entre sí, entre otras (Prieto Navarro, 2007).

De ahí que este último deba desarrollar, a su vez, habilidades emocionales para identificar, comprender y regular sus propias emociones, ya que estas han demostrado una incidencia en los procesos de aprendizaje, en la salud física, en la calidad de las relaciones interpersonales y en el rendimiento académico del estudiantado, así como en el desempeño laboral del personal docente (Cabello, Ruiz-Aranda y Fernández-Berrocal, 2010).

Junto con lo dicho, es necesario que existan canales de comunicación y la acción coordinada entre el personal docente, las madres y padres de familia, a fin de que el trabajo realizado tenga continuidad, pues, como hemos señalado, los contextos educativo y familiar son los principales protagonistas en la educación emocional de los niños y adolescentes (Márquez-Cervantes y Gaeta-González, 2018).

Si partimos de la idea de que el ambiente familiar y el escolar son los que más influyen en el desarrollo del individuo y en su proceso educativo, resulta necesaria la colaboración coordinada de estos actores en la formación de los niños y de los adolescentes. La carencia del trabajo conjunto entre las familias y el ámbito educativo puede provocar que nuestra sociedad genere personas frustradas, violentas e irresponsables, víctimas de adicciones e inseguridad. Es por esto que resulta fundamental fortalecer el desarrollo emocional de los niños y adolescentes de manera de facilitar su tránsito hacia una vida adulta con equilibrio y satisfacción (Márquez Cervantes y Gaeta González, 2018). 


\section{METODOLOGÍA Y PROCEDIMIENTOS}

\subsection{DISEÑO METODOLÓGICO}

El trabajo adoptó un enfoque cuantitativo, con la finalidad de analizar la realidad estudiada con datos numéricos y estadísticos. En este contexto, se optó por utilizar un diseño metodológico de carácter correlacional y descriptivo, con la finalidad de conocer y describir la relación existente entre las variables de estudio (Hernández Sampieri, Fernández Collado y Baptista Lucio, 2010).

Se plantearon dos hipótesis:

- Existen diferencias en cómo se percibe el ambiente escolar en función de la etapa del ciclo vital.

- La percepción del soporte y del apoyo brindado por los padres varía en función del rol que se desempeña en la institución escolar.

\subsection{MUESTRA}

Se conformó una muestra no probabilística y por conveniencia, integrada por 157 personas que forman parte de un colegio público de la ciudad de San Juan (Argentina). Entre estas, 134 eran alumnos del nivel secundario, y 23 ocupaban cargos docentes y directivos en el mismo nivel de enseñanza. A su vez, en el caso de los primeros, su promedio de edad era de 15,67 años, mientras que en el de los segundos, de 42,05 años; entre ellos, el 47,8\% eran hombres y el $52,2 \%$ mujeres.

\subsection{INSTRUMENTO SELECCIONADO}

El instrumento seleccionado fue la Escala sobre el perfil solidario de la comunidad educativa - II. School as a Caring Community Profile Scale - II (SCCP-II) de Lickona y Davidson (2003). ${ }^{3}$

El inventario está dirigido a evaluar la percepción que los integrantes de una institución escolar poseen sobre la cultura solidaria que en esta predomina. Posee un formato de escala de respuestas tipo Likert, con cinco alternativas de respuesta y 42 ítems, que se distribuyen en cinco subescalas claramente identificadas (Lickona y Davidson, 2003). Las tres primeras, están dirigidas en forma directa a evaluar la percepción de los alumnos:

1) Subescala IA. "Percepción de respeto" que explora cómo los estudiantes se cuidan, estiman y evitan maltratar tienen entre sí4;

2) Subescal IB. "Percepción de amistad y sentido de Pertenencia", mide los vínculos afectivos, la convivencia y el sentimiento de unidad que prevalece entre los alumnos;

Junto con este, se realizaron preguntas sociodemográficas para recoger información referente al sexo, la edad y el rol que los sujetos desempeñan en la institución educativa.

$4 \quad$ Algunos de los ítems que la integran son: "1. Los estudiantes tratan a sus compañeros respetuosamente"; "9. Los estudiantes tratan respetuosamente a los que trabajan en la escuela".

5 Ejemplos de ítems: "5. Los estudiantes se ayudan unos a otros, incluso cuando no son amigos"; "13. Los estudiantes ayudan a que sus nuevos compañeros se sientan aceptados en el grupo". 
3) Subescala IC. "Percepción de la configuración del medioambiente", evalúa el clima que se genera en la institución escolar a partir de las interacciones que se desarrollan entre los alumnos y de cómo estos respetan las normas establecidas ${ }^{6}$.

Las dos últimas, se refieren a la percepción de la institución escolar, de los actores que la conforman, y de las familias:

4) Subescala IIA. "Percepción del soporte recibido y brindado por la institución", mide el respeto que el personal docente, directivo y administrativo se tiene entre sí y expresa a los alumnos y a los padres de familia ${ }^{7} ; \mathrm{y}$

5) Subescala IIB. "Percepción del soporte recibido y brindado por los padres", mide el respeto que los progenitores expresan hacia la comunidad escolar, en particular a los docentes, directivos y otros padres de familia; el respeto que los docentes tienen por los padres; y la coherencia que existe entre los valores y las virtudes transmitidos en la escuela y en la familia ${ }^{8}$.

En su estudio original (Lickona y Davidson, 2003) la confiabilidad del instrumento se realizó a través del alpha de Cronbach, cuyo análisis arrojó los siguientes resultados:

- En la Subescala IA: total de la población estudiada: 0,83; en la población adolescente: 0,75 ; y en la población adulta: 0,88 .

- En la subescala IB: total de la población estudiada: 0,85; en la población adolescente: 0,81 ; y en la población adulta: 0,87 .

- En la Subescala IC.: total de la población estudiada: 0,87; en la población adolescente: 0,85 ; y en la población adulta: 0,88 .

- En la Subescala IIA: total de la población estudiada: 0,80; población adolescente: 0,79 y en la población adulta: 0,73 .

- En la Subescala IIB: total de la población estudiada: 0,70; en la población adolescente: 0,69; y en la población adulta: 0,70.

\subsection{PROCEDIMIENTO}

Se solicitó el permiso correspondiente a las autoridades del colegio que participó en la investigación; de la misma manera, a los alumnos y personal docente y directivo que completaron los cuestionarios, se les brindó información sobre los objetivos del trabajo, el carácter confidencial y voluntario de su participación.

El tiempo empleado para completar los instrumentos varió entre 40 y 60 minutos.

Los puntajes de la Escala sobre el perfil solidario de la comunidad educativa (SCCP, para sus siglas en inglés) se obtuvieron sumando las elecciones de los sujetos y convirtiendo la suma a escala 10. Los datos fueron procesados mediante la utilización del programa SPSS -Statistical Package for the Social Sciences - versión 23.0.

Ejemplos de ítems: "6. Cuando hacen algo malo, los estudiantes intentan arreglar el daño"; "8. Los estudiantes ayudan a sus compañeros a seguir las reglas de la escuela".

$7 \quad$ Algunos ítems que conforman la subescala son: "26. Los estudiantes pueden hablar con sus profesores sobre los problemas que les preocupan o inquietan"; " 38 . Todo el personal de la escuela se trata con respeto".

$8 \quad$ Ejemplos de algunos ítems que integran la escala: "27. Los padres, madres o tutores muestran interés por la educación de sus hijos y por su comportamiento en la escuela"; "42. Los padres, madres o tutores participan activamente en la escuela". 


\subsection{ANÁLISIS E INTERPRETACIÓN DE LOS RESULTADOS}

\subsubsection{Análisis descriptivo}

Se realizó un análisis descriptivo (Tabla 1) cuyos resultados expresan que en la muestra predomina, en la Institución, una percepción positiva sobre el soporte brindado por los padres de familia $(X=7,97)$, lo que redundaría en una mayor coherencia con respecto a los valores transmitidos en la escuela y en el sistema familiar. Del mismo modo, se destaca el puntaje referente a la precepción del soporte brindado por la institución $(X=7,22)$, efecto que repercutiría en el desarrollo de un clima interno y externo positivo, que se manifestaría en sentimientos favorables entre los docentes y los directivos, como así también entre los padres y la institución que permitirían afirmar que unos y otros se sienten conformes por el sostén recibido y brindado, lo que contribuiría en el aumento del sentido de pertenencia institucional.

Llama la atención el puntaje alcanzado en la subescala IC. Sobre "Percepción de la configuración del medioambiente" $(\mathrm{X}=5,72)$ y que expresa que los alumnos tendrían dificultades para acatar las normas establecidas por el colegio, trabajar en equipo, resolver conflictos en forma pacífica y para efectuar acciones que ayuden a producir mejoras en la institución escolar. Probablemente, entre los factores causales de este resultado, es la edad en la que se encuentra la mayoría de los sujetos que participaron en el estudio, que son adolescentes.

Tabla 1. Análisis descriptivo de las subescalas que integran la Escala sobre el perfil solidario de la comunidad educativa

\begin{tabular}{|l|c|c|c|c|c|}
\hline Subescala & N & Mínimo & Máximo & Media & Desv. típ. \\
\hline Subescala IA. Percepción de respeto & 157 & 2.50 & 9.50 & 5.85 & 1.10 \\
\hline $\begin{array}{l}\text { Subescala IB. Percepción de amistad } \\
\text { y sentido de pertenencia }\end{array}$ & 157 & 3.25 & 10.00 & 7.05 & 1.39 \\
\hline $\begin{array}{l}\text { Subescala IC. Percepción de la } \\
\text { configuración del medioambiente }\end{array}$ & 157 & 2.29 & 10.00 & 5.72 & 1.44 \\
\hline $\begin{array}{l}\text { Subescala IIA. Percepción del soporte } \\
\text { recibido y brindado por la institución }\end{array}$ & 157 & 2.67 & 8.89 & 7.22 & 1.21 \\
\hline $\begin{array}{l}\text { Subescala IIB. Percepción del soporte } \\
\text { recibido y brindado por los padres }\end{array}$ & 157 & 4.29 & 10.00 & 7.97 & 1.16 \\
\hline
\end{tabular}

Fuente. Elaboración propia. 


\subsubsection{Comparación entre las subescalas que integran el instrumento y algunas variables demográficas}

A fin de corroborar la existencia de diferencias estadísticamente significativas entre las subescalas que conforman el SCCP-II y algunas variables demográficas, como el sexo, la etapa del ciclo vital y el rol desempañado en la institución educativa, se efectuaron sucesivos análisis multivariante de varianza (MANOVA). En todos, como variables dependientes se consideraron las subescalas que integran el instrumento $\mathrm{y}$, como variables independientes los aspectos sociodemográficos.

Con respecto al análisis efectuado entre las subescalas del SCCP-II y el sexo, si bien no se encontraron diferencias estadísticamente significativas, las mujeres manifiestan tener una visión más positiva sobre el perfil solidario de la comunidad escolar, con excepción del soporte hacia y desde los padres, al que perciben más negativamente (gráfico 1).

Gráfico 1. Comparación de los valores medios alcanzados en las subescalas del SCCP-II en función del sexo

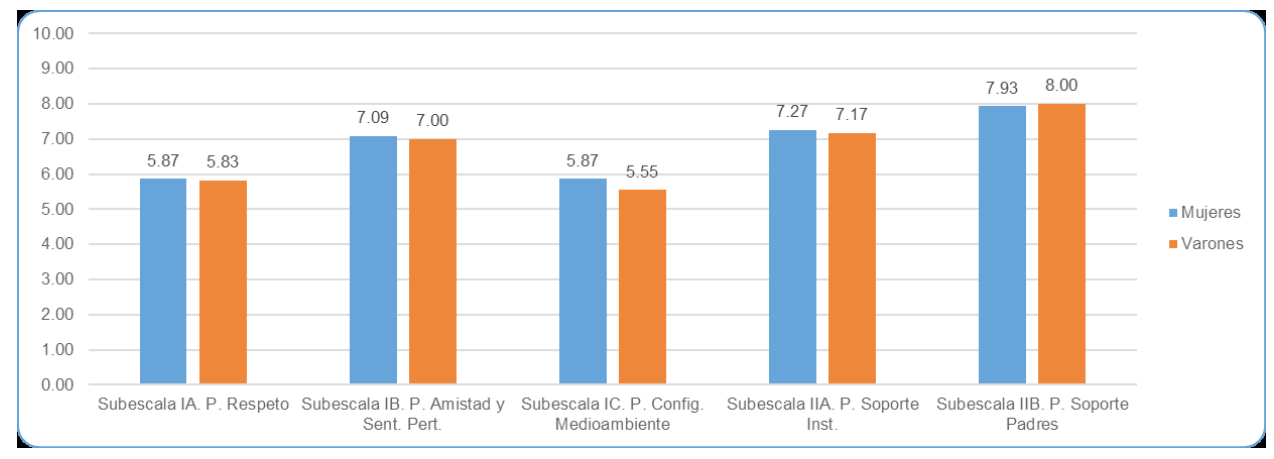

Fuente. Elaboración propia.

Para efectuar el mismo análisis en función de la etapa del ciclo vital, se optó por dividir la muestra en dos subgrupos que se denominaron "Adolescencia" y "Adultez", integradas por 134 y 23 sujetos respectivamente.

El MANOVA mostró una asociación entre las subescalas del test y la etapa del ciclo vital (adolescentes y adultos); en efecto, el criterio de Lambda de Wilkis muestra cómo las subescalas del cuestionario son afectadas en forma significativa según la etapa de la vida [Wilks' $\lambda=0,750, \mathrm{~F}(5,151.000)=10,070, \mathrm{p}<0,0001$, varianza explicada 25\%]. Dada la importancia del análisis, se examinaron los efectos principales univariados, fruto de lo cual se obtuvieron efectos estadísticamente significativos entre la subescala IIB "Percepción del soporte recibido y brindado por los padres" y etapa del ciclo vital, ( $\mathrm{F}=14,640, \mathrm{p}<0,001$, varianza explicada 96\%), que favorecen a la muestra más joven. 
Gráfico 2. Comparación de los valores medios alcanzados en las subescalas del SCCP-II en función de la etapa del ciclo vital

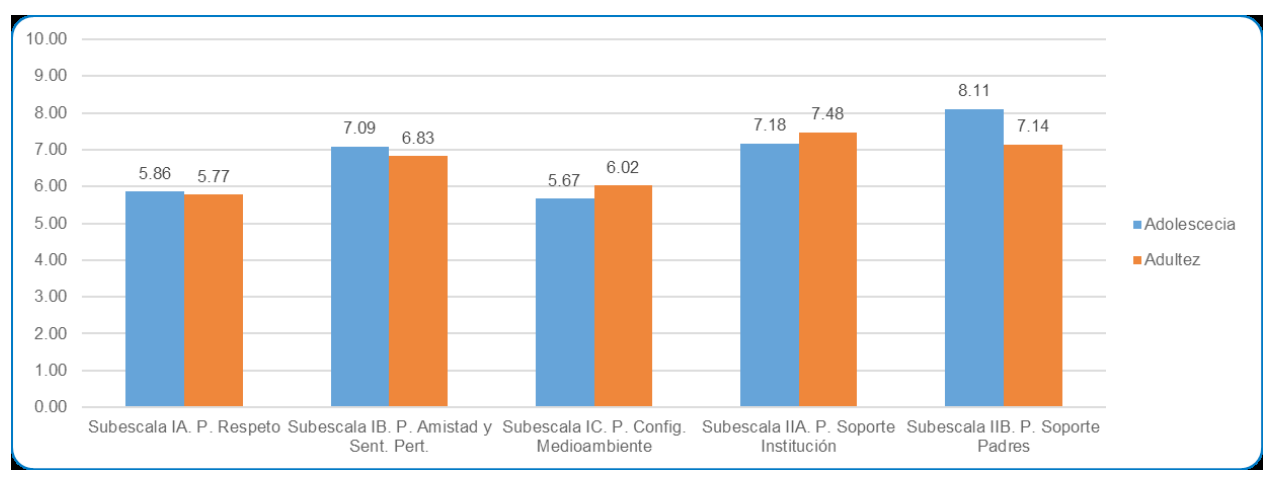

Fuente. Elaboración propia.

Para realizar el MANOVA teniendo en cuenta el rol desempeñado en la institución como variable dependiente, se consideró dividir la muestra en otros dos grupos: estudiantes, y personal docente y directivo.

Se encontró una vinculación entre las subescalas del cuestionario y el rol ejercido por los actores institucionales; efectivamente, el criterio de Lambda de Wilkis evidencia cómo las subescalas son afectadas en forma significativa por el rol ejercido [Wilks' $\lambda=0,750$, $\mathrm{F}(5,151.000)=10,070, \mathrm{p}<0,0001$, varianza explicada $25 \%$ ]. En este caso también, se exploraron los efectos principales univariados, fruto de lo cual se obtuvieron diferencias estadísticamente significativas entre rol desempeñado y la subescala IIB "Percepción del soporte recibido y brindado por los padres" ( $\mathrm{F}=14,640, \mathrm{p}<0,001$, varianza explicada $96 \%$ ) que beneficia a los alumnos.

Gráfico 3. Comparación de los valores medios alcanzados en las subescalas del SCCP-II en función del rol desempeñado en la institución

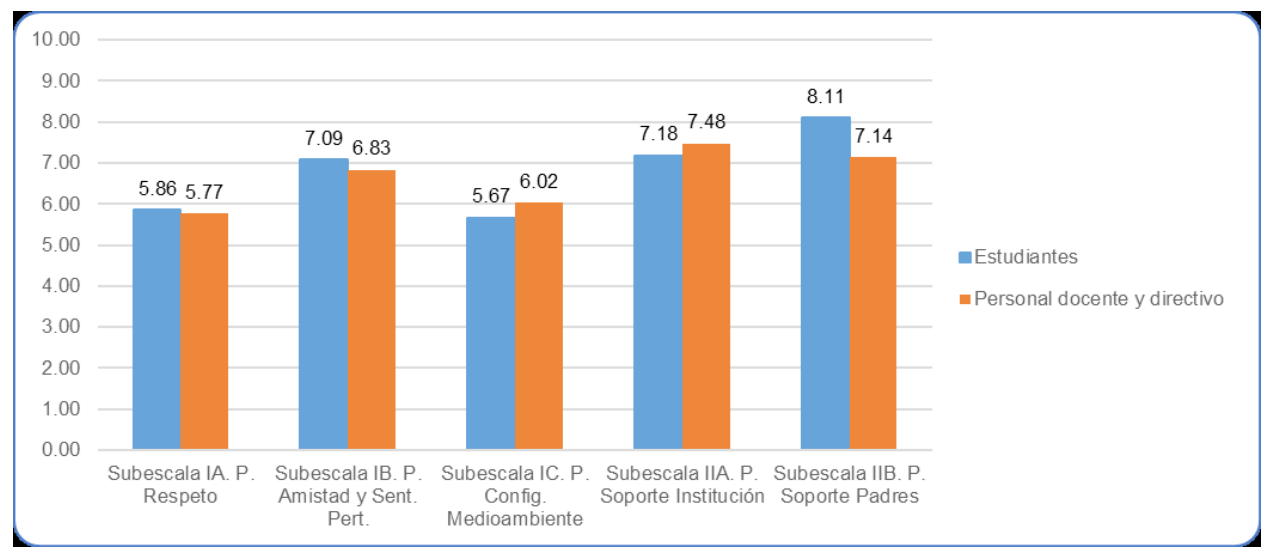

Fuente. Elaboración propia. 


\section{DISCUSIÓN Y CONCLUSIONES}

El estudio de la educación socioemocional y de la comunidad escolar como contexto que puede propiciar o no su desarrollo, ocupan un lugar preponderante en el ámbito académico y educativo.

A través de las líneas aquí desarrolladas se remarca la importancia de promover una educación integral que contemple la promoción de la dimensión social y emocional de cada educando, más aún si se considera que, en los primeros años de escolaridad, se consolidad la personalidad, se priorizan valores en coherencia con un proyecto de vida al que se elige y se consolidan virtudes o fortalezas de carácter. Es en ese contexto, en integración con el ámbito familiar y social, en el que la persona va dando sus primeros pasos como ciudadano de la sociedad de la cual forma parte.

En línea con estas ideas, los resultados obtenidos a través de la aplicación de instrumento School as a Caring Community Profile Scale - ii (SCCP-II), dirigido a evaluar cómo se percibe el ambiente solidario de la comunidad educativa, muestran que existen diferencias de acuerdo con la edad y con el rol que se ocupa en la institución educativa en la que se realizó el estudio.

En este sentido, los sujetos más jóvenes en edad, que también ocupan el rol de estudiantes son quienes alcanzaron los resultados más positivos que indican que, en general, perciben que en la escuela predomina un ambiente de respeto entre los compañeros, que existen vínculos de amistad sanos y fuertes, como así también que predomina un clima institucional agradable y respetuoso.

En paralelo, esta misma población, considera que existe un vínculo respetuoso entre el personal docente, directivo y administrativo, que se proyecta hacia el alumnado y al sistema familiar; efecto que incidiría a que los padres traten con mayor consideración y sentido de autoridad a los docentes y directores del colegio.

Con respecto al menor puntaje alcanzado por estos últimos sujetos, pueden efectuarse varias hipótesis asociadas a la desmotivación generalizada que predomina en los profesionales de la enseñanza de la Argentina y que necesariamente impacta en su desempeño laboral (Menghi y Oros, 2014); como así también al predominio de docentes que se desempeñan en varias instituciones educativas en forma simultánea, lo que repercute negativamente en su nivel de rendimiento (Murillo Tordecilla y Román Carraso, 2013).

Sin duda, los resultados recabados permiten considerar algunas estrategias que podrían implementarse en la institución educativa para fortalecer la percepción optimista que los alumnos tienen sobre el ambiente solidario que en ella predomina, y para favorecer que este mismo efecto se acreciente en el personal docente y directivo.

Las primeras estrategias a considerar se orientan a atender al personal que se desempeña laboralmente en la institución, partiendo de la premisa de que, si no existe un clima favorable entre ellos y un sentimiento de pertenencia hacia el colegio, a corto, mediano o largo plazo, este efecto se reflejará en el trabajo que se realiza con los alumnos.

En coherencia con este planteo, pueden planificarse entrevistas personales de seguimiento, llevadas a cabo por el personal directivo y orientadas a incrementar la motivación intrínseca y trascendente ${ }^{9}$ a fin de conocer cuáles son sus intereses personales y profesionales, cuáles de entre éstos pueden enriquecer la labor pedagógica que se

Pueden consultarse las obras de Nuttin (1980) y de Pérez López (1996) y vincular su temática con el ámbito educativo. 
lleva a cabo en la institución, cuáles son aquellos que los ayudan a salir de sí mismos, y a orientarlos a que redescubran el propósito que tienen tanto la comunidad educativa como su labor personal dentro de esta.

Las entrevistas de seguimiento personal pueden complementarse con reuniones de equipo orientadas a profundizar el desarrollo de la dimensión socioemocional en el proyecto educativo institucional, en general, y en cada estudiante, en particular.

Estas reuniones de equipo pueden planificarse en orden a efectuar una revisión del diseño curricular institucional, en coherencia con el diseño curricular juridiccional, para incluir en éste el desarrollo de competencias o capacidades socioemocionales en los alumnos, respetando su etapa evolutiva y nivel educativo de pertenencia.

Las segundas estrategias a implementar, dirigidas a los alumnos, pueden incluir la organización y planificación de tutorías personales o grupales, a través de las cuales, se aborden contenidos vinculados con las capacidades ya mencionadas.

El espacio que se genere a través de las tutorías tiene que complementarse con el trabajo que se realice en conjunto con los padres y madres de familia, en cuyo ámbito se inicia el desarrollo socioemocional de cada alumno. En efecto, para favorecer una mayor consciencia por parte de estos en cuanto a su rol primordial como primeros educadores, desde la escuela es conveniente que se generen espacios de formación dirigidos tanto a los progenitores como a los docentes y en los que se aborden temáticas vinculadas con la dimensión social y emocional del educando; entre ellas: el desarrollo de un proyecto vital a partir del autoconocimiento y de la sana autoestima; la promoción de fortalezas de carácter o de capacidades vinculadas a la dimensión cognitiva (como por ejemplo, la creatividad, la curiosidad, el amor por el aprendizaje), a la dimensión afectivo motivacional (perseverancia, integridad, amor, autorregulación emocional, etc.), a la dimensión trascendente (ciudadanía, liderazgo, solidaridad, etc.).

El desarrollo de estas estrategias, como así también la realización del presente estudio en otros contextos, ayudará a incrementar el estudio de esta temática en el contexto educativo argentino.

De acuerdo con las ideas expuestas, el presente trabajo constituye un aporte para continuar fomentando el desarrollo de competencias socioemocionales en el alumnado dentro del contexto escolar, en coordinación con los padres y madres de familia, ya que tomar consciencia de las propias emociones y adquirir herramientas para gestionarlas adecuadamente, contribuye a tomar decisiones responsables y a promover una convivencia armónica, previniendo situaciones de riesgo y violencia. En definitiva, se trata que, desde la escuela, se favorezca la construcción de una sociedad con menos violencia, menos estrés, y con mayor seguridad, satisfacciones y bienestar para las futuras generaciones.

El personal docente reporta el trabajo colaborativo con las familias como un medio para el desarrollo integral de los chicos y chicas. No obstante, existen áreas de oportunidad para fomentar la cooperación entre las familias y los centros escolares, que permitan coordinar esfuerzos hacia el logro de los mismos objetivos, con una responsabilidad compartida y coordinada, hacia una educación emocional de los adolescentes.

Como hemos señalado, el desarrollo emocional no es una labor solo del centro educativo, sino principalmente de los padres y madres de familia; ya que es parte de un aprendizaje que se inicia y se vive en la familia y se complementa en la escuela. De ahí la importancia de que los progenitores sean conscientes y contribuyan al desarrollo de competencias emocionales en sus hijos y en sí mismos como un aspecto esencial de su formación básica y primordial. 
El presente estudio constituye un aporte para seguir fomentando el desarrollo de competencias emocionales en el alumnado dentro del contexto escolar, en coordinación con los padres y madres de familia, ya que el tomar consciencia de las propias emociones y adquirir herramientas para gestionarlas adecuadamente, contribuye a tomar decisiones responsables y promueve una convivencia armónica, para prevenir situaciones de riesgo y violencia. Se trata, en definitiva, de contribuir desde la escuela a la construcción de una sociedad con menos violencia, menos estrés, y con mayor seguridad, satisfacciones y bienestar para las futuras generaciones.

\section{REFERENCIAS BIBLIOGRÁFICAS}

Alterio Arriola, F. y Pérez Loyo, F. (2005). Inteligencia Emocional: Teoría y Práctica en Educación. Universidad Centroccidental "Lisandro Alvarado".

Amilbiru, Ma. G. (2015). ¿Competencias, capacidades o virtudes? Matices importantes en el lenguaje educativo. Pedagogia e Vita, 73, 37-52.

Bar-On, R. (1997). The Bar-On Emotional Quotient Inventory (EQ-i): A test of emotional intelligence. Toronto: Multi-Health Systems.

Barrio Maestre, J. Ma. (2016). Homo Adulescens: elementos para una teoría antropológica de la educación. Buenos Aires: Teseo Press.

Beech, J. y Marchesi, A. (2008). Estar en la escuela, estudio sobre convivencia. Buenos Aires: OEI. Recuperado el 4 de julio de 2012 de: http://www.oei.es/valores2/EstarenlaEscuela1.pdf

Berger, K. (2016). Psicología del desarrollo: infancia y adolescencia. Madrid: Médica Panamericana S.A.

Berger, C., Milicic, N., Alcalay, L., Torretti, A., Arab, Ma. P. y Justiniano, B. (2009). Bienestar socio-emocional en contextos escolares: la percepción de estudiantes chilenos. Estudios sobre Educación, (17), 21-43. Consultado en https://www.unav.edu/publicaciones/revistas/index.php/ estudios-sobre-educacion/article/viewFile/22422/18651.

Berkowitz, M. (2018). Fundamentals of Effective Character Education. Character Education in Latin America: Challenges and Opportunities. Austral University, Pilar Campus (Argentina). June 1314,2018

Bernal, A., González-Torres, M. C. y Naval, C. (2015). La Educación del carácter. Perspectivas internacionales. Participación educativa segunda época, 4(6), 35-45. Retrieved from: https:// sede.educacion.gob.es/publiventa/la-educacion-del-caracter-perspectivas-internacionales/ educacion-politica-educativa/20413.

Bernard, B. (2004). Resiliency: What we have learned. San Francisco, CA: WestEd.

Cabello, R., Ruiz-Aranda, D. \& Fernández-Berrocal, P. (2010). Docentes emocionalmente inteligentes. Revista Electrónica Interuniversitaria de Formación del Profesorado, 13(1), 4149. Retrieved from: http://www.redalyc.org/articulo.oa?id=217014922005.

Cáceres Muñoz, J. (2015). La escuela de madres y padres como escuela de emociones. Un impulso hacia la colaboración en beneficio de la calidad educativa. Investigar con y para la sociedad, (2), 637-646. Retrieved from http://aidipe2015.aidipe.org.

Castillo Córdova, G. (2013). La normalidad afectiva en la educación de los hijos. Estudios sobre Educación, (25), 151-166.

Çınkır, S., Nayir, K. \& Çetin, S. (2016). The Adaptation of Scale of School as a Caring Community Profile for Secondary School Students into Turkish: Adaptation of School as a Caring Community Scale. International Journal of Higher Education, 5(4). doi:10.5430/ijhe.v5n4p206.

Clouder, I., Ma. Mikuli, I., M. Leibovici-Mühlberger; E. Yariv; J. Finne y P. Van Alphen. (2013). Educación Emocional y Social. Análisis Internacional (pp. 131-76). Santander: Fundación Botín. 
Estudios Pedagógicos XLV, $\mathrm{N}^{\circ}$ 3: 31-49, 2019

LA COMUNIDAD ESCOLAR COMO MEDIO PARA EL DESARROLLO SOCIO EMOCIONAL DE LOS ALUMNOS.

UN ESTUDIO DE CASO EN LA REPÚBLICA ARGENTINA

Consultado en https://www.observatoriodelainfancia.es/ficherosoia/documentos/4043_d_ Educacion_Emocional_Botin_2013.pdf.

Craig, G. (2009). Desarrollo psicológico. México: Editorial Pearson.

Cuervo Martínez, Á. (2010). Pautas de crianza y desarrollo socioafectivo en la infancia. Perspectivas en Psicología, 6(1), 111-121. Retrieved from http://www.redalyc.org/articulo.oa?id=67916261009.

De Fabritis, V. N. (julio de 2018). Programa de Educación Emocional pionero en la Argentina basado en la Cultura del Pensamiento (Universidad de Harvard). Congreso Internacional de Educación y Aprendizaje. Université Paris Diderot, Paris, Francia.

Dweck, C. S. y Leggett, E. L. (1988). A social-cognitive approach to motivation and personality. Psychological Review, 95(2), 256-273.

Gaeta, M. L. \& López, C. (2013). Competencias emocionales y rendimiento académico en estudiantes universitarios. Revista Electrónica Interuniversitaria de Formación del Profesorado, 16(2), 13-25.

Gaeta González, M. L. \& Martínez-Otero Pérez, V. (2017). Las competencias emocionales en la educación formal. Reflexiones y experiencias de investigación en diferentes contextos educativos. Revista Iberoamericana de Educación, 77(2), 179-183.

Garrido Natarén, P. \& Gaeta González, M. L. (2016). La competencia socioemocional docente en el logro del aprendizaje de las competencias genéricas del perfil de egreso de educación media superior. Revista de Comunicación Vivat Academia, 19(137), 108-123. doi:https://doi. org/10.15178/va.2016.137.108-123.

Goleman, D. (2012). Inteligencia emocional. Barcelona: Ed. Kairós.

Henao López, G. C. \& García Vesga, M. C. (2009). Interacción familiar y desarrollo emocional en niños y niñas. Revista Latinoamericana de Ciencias Sociales, Niñez y Juventud, 7(2), 785-802.

Hernández-Veloz, M. J., Gaeta-González, M. L. \& García-Gordillo, S. (2016). Variables sociofamiliares y afectivo-motivacionales en relación al rendimiento académico en Educación Media Superior. Cuadernos Hispanoamericanos de Psicología, 16(2), 5-74.

Hero (2016). Hero Intervention. Buenos Aires: https://herointervention.com

Hernández Sampieri, R., Fernández-Collado, C. y Baptista Lucio, P. (2010). Definición conceptual o constitutiva. ( $5^{\mathrm{a}}$ ed.). México: McGraw-Hill.

Lickona, D. \& Davidson, M. (2003). School as a caring community profile-II. Unpublished manuscript, Center for the 4th and 5th Rs, Cortland.

Litichever, L. (2012). ¿Qué se regula hoy en las escuelas? Una mirada sobre las prescripciones de los reglamentos de convivencia. Revista Iberoamericana de Educación, 59(1). 1-10.

Márquez Cervantes, M. C. (2017). Adolescencia, una oportunidad para la educación de las emociones. En: M. L., Gaeta González y V. Martínez-Otero Pérez (coords.). Las competencias emocionales en la educación formal. Reflexiones y experiencias de investigación en diferentes contextos educativos. Ciudad de México, Universidad Popular Autónoma del Estado de Puebla.

Márquez Cervantes, M. C. \& Gaeta González, M. L. (2016). La educación emocional y el trabajo de los padres de familia. En: M. Pérez-Fuentes, J. J. Gázquez Linares, M. Molero Jurado, Á. M. Martínez, Ma del Mar Simón, A. B. Barragán Martín (coords.). Variables Psicológicas y Educativas para la intervención en el ámbito escolar, II. España. ASUNIVEP.

. (2018). Competencias emocionales y toma de decisiones responsable en preadolescentes con el apoyo de docentes, padres y madres de familia: Un estudio comparativo en estudiantes de $4^{\circ}$ a $6^{\circ}$ año de educación primaria en España. Revista Electrónica Educare, 22(1), 1-25. doi:http://dx.doi.org/10.15359/ree.22-1.9

Martínez-Garrido, C. \& Murillo, F. (2015). Desarrollo del autoconcepto de los estudiantes en la escuela: un estudio de los factores asociados. En AIDIPE (ed.), Investigar con y para la sociedad, 2, 911-921. Cádiz, España: Bubok. Recuperado de http://aidipe2015.aidipe.org

Menghi, Ma. S. y Oros, L. B. (2014). Satisfacción laboral y Síndrome de Burnout en docentes de nivel primario. Revista de Psicología, 10(20), 47-59.

Mesurado, B., Disfefano, Ma. J., Robiolo, G. y Richaud, Ma. C. (2018). The Hero program: 
Development and initial validation of an intervention program to promote prosocial behavior in adolescents. Journal of Social and Personal Relationships, 1-20. Consultado en: https:// www.researchgate.net/publication/326304982_The_Hero_program_Development_and_initial_ validation_of_an_intervention_program_to_promote_prosocial_behavior_in_adolescents.

Mikulic, I. Ma. (2013). La educación emocional y social en Argentina: entre certezas y esperanzas. En C. Clouder, I. Ma. Mikulic, M. Leibovici-Mühlberger, E. Yariv, J. Finne y P. Van Alphen. Educación Emocional y Social. Análisis Internacional, 131-76. Santander: Fundación Botín. Consultado en https://www.observatoriodelainfancia.es/ficherosoia/documentos/4043_d_ Educacion_Emocional_Botin_2013.pdf

Montoya-Castilla, I., Prado-Gascó, V., Villanueva-Badenes, L., \& González-Barrón, R. (2016). Adaptación en la infancia: influencia del estilo parental y del estado de ánimo. Acción Psicológica, 13(2), 15-30.

Murillo Tordecilla, F. J. y Román Carraso, M. (2013). Docentes en educación primaria en América Latina con más de una actividad laboral: situación e implicaciones. Revista Mexicana de Investigación Educativa, 18(58), 893-924. Consultado en http://www.scielo.org.mx/scielo. php?script=sci_arttext\&pid=S1405-66662013000300010\&lng=es\&tlng=es

Naval, C., Bernal, A. y Fuentes, J. L. (2018). Educación del carácter y de las virtudes. En Diccionario Interdisciplinar Austral, editado por Claudia E. Vanney, Ignacio Silva y Juan F. Franck. URL: http://dia.austral.edu.ar/Educación_del_carácter_y_de_las_virtudes.

Nicholls, J. G. (1978). The development of the concepts of effort and ability, perceptions of attainment and the understanding that difficult tasks require more ability. Child development, (49), 800-814. . (1984). Achievement motivation: Conceptions of ability, subjective experience, task choice and performance. Psychological Review, (21), 328-346.

(1989). The competitive ethos and democratic education. Cambridge, MASS: Harvard University Press.

Nuttin, J. (1980). Teoría de la motivación humana. Barcelona: Paidós.

Paneiva Pompa, J. P., Bakker, L. y Rubiales, J. (2016). Características socio-emocionales del contexto de enseñanza y aprendizaje: estudio del clima social escolar en instituciones educativas de la ciudad de Mar del Plata. Anuario de Proyectos e Informes de Becarios de Investigación, (13), 1505-1520. Consultado en http://www.mdp.edu.ar/psicologia/psico/investigacion/filesinves/ anuario2016.pdf\#page $=128$

Park, N., Peterson, C. y Seligman, M. E. P. (2004). Strengths of character and well-being. Journal of Social and Clinical Psychology, 23(5), 603-619.

Peterson, C. \& Seligman, M. E. (2004). Character strengths and virtues: A handbook and classification. New York: Oxford University Press and Washington, DC: American Psychological Association.

Prieto Navarro, L. (2007). Autoeficacia del profesor universitario: Eficacia percibida y práctica docente. España: Narcea Ediciones.

Reina Flores, C. \& Oliva, A. (2015). De la competencia emocional a la autoestima y satisfacción vital en adolescentes. Behavioral Psychology / Psicología Conductual, 23(2), 345-359. Retrieved from https://www.researchgate.net/publication/285199595.

Rodríguez Fernández, T. \& Linares Von Schmiterlow, C. (2002). Enseñar y convivir: Hacia una educación dialogada. Revista Electrónica Interuniversitaria de Formación del Profesorado, 5(3). Retrieved from: http://web.archive.org/web/20041216093815/www.aufop.org/publica/reifp/ articulo. asp?pid=210\&docid $=916$.

Salovey, P. y Mayer, J. D. (1990). Emotional intelligence. Imagination, Cognition, and Personality, (9), 185-211.

Shapiro, L. E. (1997). La inteligencia emocional de los niños. Buenos Aires: Vergara Editor.

Trianes Torres, M. V. \& García Correa, A. (2002). Educación socio-afectiva y prevención de conflictos interpersonales en los centros escolares. Revista Interuniversitaria de Formación del Profesorado, (44), 175-189. 
